\title{
Evaluation of the phylogenetic position of the sulfate-reducing bacterium Thermodesulfovibrio yellowstonii (phylum Nitrospirae) by means of gene order data from completely sequenced genomes
}

\author{
Takashi Kunisawa
}

Correspondence

Takashi Kunisawa

kunisawa@rs.noda.tus.ac.jp
Department of Applied Biological Science, Science University of Tokyo, Noda 278-8510, Japan

\begin{abstract}
The phylogenetic placement of Thermodesulfovibrio yellowstonii was investigated on the basis of gene order data from completely sequenced bacterial genomes. T. yellowstonii was found to share four gene arrangements characteristic of the Proteobacteria, Aquificae, Planctomycetes, Spirochaetes, Bacteroidetes, Chlorobi, Acidobacteria, Verrucomicrobia and termite group 1, the presence of which defines superphylum 2. The remaining phyla show sets of alternative gene arrangements and form superphylum 1. An analysis of conserved gene pairs showed that the overall genome organization of $T$. yellowstonii is most similar to that of deltaproteobacteria. Three arrangements that suggest gene translocations were identified that are likely to have occurred in a common ancestor of $T$. yellowstonii and the Proteobacteria exclusive of virtually all other major bacterial phyla. The translocation events suggest the closest evolutionary relationship between $T$. yellowstonii and the Proteobacteria.
\end{abstract}

\section{INTRODUCTION}

Thermodesulfovibrio yellowstonii is a thermophilic, sulfatereducing bacterium that was originally isolated from thermal vent water in Yellowstone Lake (Henry et al., 1994). An early phylogenetic study based on $16 \mathrm{~S}$ rRNA gene sequence comparisons indicated that the Gram-negative $T$. yellowstonii branched deeply in the domain Bacteria and is classified in a distinct phylum, Nitrospirae (Henry et al., 1994). The placement as the third deepest branch, following two other thermophiles, Aquifex and Thermotoga, supports the theory of thermophilic origins of the Bacteria (Achenbach-Richter et al., 1987). A more recent analysis of $16 \mathrm{~S}$ rRNA gene sequences indicated that the phylum Nitrospirae, including T. yellowstonii, branched off after the divergence of Aquifex, Thermotoga, Chloroflexus and the Thermus-Deinococcus group and then formed a cluster with proteobacteria (Rhodospirillum rubrum and Chromatium vinosum) and a Gram-positive bacterium, Bacillus subtilis (Castro et al., 2000). On the other hand, another member of the phylum Nitrospirae, Nitrospira marina, was reported to be affiliated with the Deltaproteobacteria (Teske et al., 1994) on the basis of $16 \mathrm{~S}$ rRNA gene sequence comparison.

Although the phylogenetic position of T. yellowstonii has so far been inferred based on the $16 \mathrm{~S}$ rRNA gene sequence, the

A list of completely sequenced bacterial genomes used in this study and results of BLAST best-hit analyses of ribosomal proteins are available as supplementary material with the online version of this paper. availability of genome sequence data (R. J. Dodson, A. S. Durkin, M. Wu, J. Eisen and G. Sutton, unpublished NCBI accession no. NC_011296; submitted 29 August 2008) presents new phylogenetic challenges. It is widely believed that genome trees based on a concatenation of multiple protein sequences are less susceptible to stochastic errors than those based on a single protein or gene such as the 16S rRNA gene. Genome trees reported recently showed the power of this approach, confirming accepted phylogenies and/or presenting unexpected novel phylogenetic relationships at the level of phyla as well as individual species (Brown et al., 2001; Wolf et al., 2001; Teeling et al., 2004; Ciccarelli et al., 2006; Wu \& Eisen, 2008; Ward et al., 2009). Concatenation of trees for individual proteins (supertree method) has also proved useful for reconstructing bacterial phylogeny in the presence of lateral gene transfer (Daubin et al., 2002). While separation of the major bacterial phyla was largely improved in genome trees or supertrees, branching order at the phylum level remained poorly resolved. Another useful approach is to find conserved insertions/deletions (indels) in protein sequences. Based on a series of conserved indels found in various proteins, a linear branching order of the major bacterial phyla, Firmicutes $\rightarrow$ Actinobacteria $\rightarrow$ Clostridium-Fusobacterium-Thermotoga $\rightarrow$ DeinococcusThermus $\rightarrow$ Chloroflexus $\rightarrow$ Cyanobacteria $\rightarrow$ Spirochaetes $\rightarrow$ Chlamydia-Cytophaga-Flavobacteria $\longrightarrow$ Chlorobi $\rightarrow$ Aquife $\longrightarrow$ Proteobacteria, has been proposed (Gupta, 1998; Gupta \& Griffiths, 2002). A third approach is to compare gene orders 
or gene contents of completely sequenced genomes (Sankoff et al., 1992; Wolf et al., 2001; Korbel et al., 2002). Although several difficulties are known in methods based on gene order or gene content (e.g. Delsuc et al., 2005), they do not rely on multiple sequence alignments and, hence, can provide independent support for phylogenies inferred from aligned sequences.

In the present paper, phylogenetic relationships of $T$. yellowstonii to other bacteria were investigated on the basis of gene arrangements that are uniquely found in a group of phyla. If a gene $x$ is located between genes $a$ and $b$, i.e. in the order $a-x-b$, in one group, while the same gene $x$ is found between genes $c$ and $d$, in the arrangement $c-x-d$, in another group, then a translocation of gene $x$ is thought to have occurred in a common ancestor of either group (Kunisawa, 2001, 2003). Three arrangements that suggest gene translocations were identified, and they provide empirical evidence that the thermophilic, sulfatereducing bacterium T. yellowstonii and the Proteobacteria are in a close, probably closest, evolutionary relationship; they share common ancestry exclusive of Acidobacteria, Chlamydiae, termite group 1, Planctomycetes, Spirochaetes and Verrucomicrobia, after the divergence from another group of phyla that comprises Firmicutes, Actinobacteria, Fusobacteria, Thermotogae, Chloroflexus, ThermusDeinococcus and Dictyoglomi.

\section{METHODS}

Gene order data. Complete genomic sequences of bacteria were downloaded from the ftp server at NCBI (ftp://ftp.ncbi.nlm.nih.gov/ genomes/Bacteria/). In order to identify orthologous relationships of genes readily among different genomes, all the protein-encoding genes were labelled systematically, using the Cluster of Orthologous Groups of proteins (COG) database, in which each homologous gene family is labelled by a unique four-digit number (Tatusov et al., 1997, 2001). Protein-encoding genes present on a genome were represented as a four-digit COG number by performing the FASTA similarity search (Pearson \& Lipman, 1988). In this assignment, a FASTA E-value of $10^{-4}$ was used as a threshold. For those genes that showed a less significant E-value, between $10^{-2}$ and $10^{-4}$, COG numbers were assigned manually by taking account of protein length, percentage identity and gene order (Kunisawa, 2006). Thus, gene order along each genome was represented as COG order. tRNA-encoding genes were searched for by the tRNAscan-SE program (Lowe \& Eddy, 1997). tRNA genes thus identified were inserted into the COG order of protein-encoding genes according to their base coordinates. rRNA genes were also put into the COG order if they were annotated in the original genomic sequence. Thus, gene order comparisons are readily carried out using the COG/ tRNA/rRNA orders thus generated. There are many completely sequenced genomes with the same species name but different strains, which reveal almost identical gene orders. Therefore, in the present analysis, one arbitrarily chosen strain was taken into account, and others were neglected. A total of 425 bacterial genomes were compared (see Supplementary Table S1, available in IJSEM Online). Although Coprothermobacter proteolyticus is annotated as a member of the Firmicutes in the NCBI taxonomy, 16S rRNA gene phylogenies indicate that this bacterium forms a distinct phylum well-separated from the Firmicutes (Etchebehere et al., 1998; Hugenholtz et al., 1998). Therefore, Coprothermobacter proteolyticus is treated as representing a separate phylum 'Coprothermobacter' in the present study.
Computer search. A computer search was carried out with an asymmetrical constraint on gene order so that $a-x-b$ could be detected in genomes of the Gram-positive actinobacteria and firmicutes and $c-x$ (or $x-d)$ in Gram-negative proteobacterial genomes and vice versa. The search procedure is simple and straightforward, as described in previous papers (Kunisawa, 2003, 2006). First, assume that one gene among the complete gene set is a transposed gene, $x$, and define its neighbouring genes, $a$ and $b$, on each genome. When an identical arrangement $a-x-b$ is found among the Actinobacteria/Firmicutes and another phylum, the other arrangement, $c-x$ (or $x-d$ ), is searched for that is conserved among the Proteobacteria and the other phyla, and vice versa. This procedure was repeated for all the genes that are present on the completely sequenced genomes. In order to avoid complicated assignments of gene orthology, the search was not carried out for cases where gene $x$ is present in multiple copies on more than half of the genomes under comparison.

\section{RESULTS}

\section{Dichotomy of bacterial phyla}

Previous analyses of gene arrangements on bacterial genomes have suggested that the major bacterial phyla are divided into two groups (superphyla 1 and 2); one group comprises the Gram-positive bacteria (Firmicutes and Actinobacteria), Thermotogae, Chloroflexi and Deinococcus-Thermus, while the other includes the Gram-negative Proteobacteria, Aquificae, Planctomycetes, Spirochaetes, Bacteroidetes and Chlorobi (Kunisawa, 2006). Since then, many more bacterial genomes have been completely sequenced, which enables phylogenomic studies of new phyla, i.e. Dictyoglomi, Acidobacteria, termite group I, Verrucomicrobia and Nitrospirae. I first verified the dichotomy of bacteria phyla in the light of the 425 currently available bacterial genomes, and then examined to which superphylum $T$. yellowstonii belongs. I carried out a computer search for sets of alternative gene arrangements, $a-x-b$ in one group of phyla and $c-x$ (or $x-d)$ in the other group for the same gene $x$. Four such arrangements were identified by the present computer search.

The first example is found around a glycine-tRNA gene with the anticodon sequence TCC (Gtcc). This Gly-tRNA gene is present in a single copy on most genomes; if present in multiple copies, its copy number per genome is shown in brackets following the species name in Fig. 1(a). Contiguous genes are connected by dashes, while split segments are interrupted by forward slashes. Homologous genes are vertically aligned. As seen in Fig. 1(a), Gtcc is located between a proline-tRNA gene with the anticodon TGG (Ptgg) and an arginine-tRNA gene with the anticodon TCT (Rtct) in 36 of 40 completely sequenced actinobacterial genomes. The numerator in parentheses following the phylum name represents the number of genomes showing conserved gene arrangements, and the denominator is the total number of compared genomes belonging to that phylum. The adjacency of Gtcc to Ptgg and/or Rtct can also be found in the Firmicutes, 
Synergistetes, Fusobacteria, Deinococcus-Thermus, Dictyoglomi and Thermotogae. Some typical gene arrangements are shown for each phylum. For example, the genome of Bacillus halodurans harbours three copies of Gtcc, two of which are adjacent to Ptgg or Rtct. Nine out of 23 genomes from the Bacillales show similar gene arrangements. By contrast, Gtcc is not found between Ptgg and Rtct in the Proteobacteria but is located between a tyrosine-tRNA gene with the anticodon sequence GTA (Ygta) and a threoninetRNA gene with the anticodon GGT (Tggt). This latter gene arrangement can be found in the Aquificae, Acidobacteria, Planctomycetes, Bacteroidetes, termite group 1 and 'Coprothermobacter' as well as T. yellowstonii (Nitrospirae). Members of the Spirochaetes, Chlorobi, Verrucomicrobia, Chlamydiae and Cyanobacteria do not reveal either of these gene arrangements. A few members of the Clostridia (Firmicutes) show a mixed arrangement; in Finegoldia magna, the Gtcc gene is located adjacent to both Rtcc and Ygta, and similar arrangements are found in Alkaliphilus metalliredigens and Alkaliphilus oremlandii, around one of the Gtcc genes present in four and five copies per genome, respectively, as shown within brackets in Fig. 1(a). Thus, except for a few clostridia, the major bacterial phyla are likely to be divided into two groups on the basis of gene arrangement around the Gtcc gene.

Similar alternative gene arrangements were identified around the nusB gene (COG0781), which encodes a transcription termination factor. In the Firmicutes, Synergistetes, Actinobacteria, Fusobacteria, DeinococcusThermus, Chloroflexi, Dictyoglomi and Thermotogae, nusB is located in the neighbourhood of efp (COG0231), yqhY (COG1302), folD (COG0190) and xseA (COG1722). In contrast, $n u s B$ is adjacent to ribH (COG0054) in T. yellowstonii and members of the Proteobacteria, Verrucomicrobia, Spirochaetes, Acidobacteria and Aquificae (Fig. 1b). In Fig. 1, for maximum alignment of gene order, some contiguous ORFs are represented by dots.

A conserved hypothetical protein-encoding gene designated $y v y D$ (COG1544) is located adjacent to comFC (COG1040) and/or to secA (COG0653) in the Firmicutes, 'Coprothermobacter', Actinobacteria, Dictyoglomi and Chloroflexi (Fig. 1c). In T. yellowstonii, yvyD is located between rpoN (COG1508) and $y h b J$ (COG1660), and similar arrangements are found in members of the Proteobacteria, termite group 1, Spirochaetes, Acidobacteria, Planctomycetes and Aquificae.

The computer search identified a fourth set of alternative gene arrangements around lepA, encoding a membrane GTPase. As shown in Fig. 1(d), the lepA gene (COG0481) is present between rpsT (COG0268) and hemN (COG0635) in the Firmicutes, Synergistetes, Actinobacteria and Dictyoglomi. In Fig. 1, genes designated with an asterisk, i.e. $r p s T$ (COG0268) and $\operatorname{deg} Q$ (COG0265), are located on the strand complementary to that of lepA, hemN and lepB, and are transcribed from right to left. In T. yellowstonii and members of the Proteobacteria, Verrucomicrobia, Bacteroidetes, Chlorobi and Cyanobacteria, the arrangement rpsT-lepA-hemN is not found, but another arrangement, $\operatorname{deg} Q$ (COG0265)-lepA (COG0481)-lepB (COG0681), is observed. Two members of the Clostridia exceptionally show a mixed arrangement, lepB-(ORF)-lepA-hemN.

The phylum-gene arrangement relationships thus obtained are summarized in Table 1 , in which gene arrangements of Gram-positive firmicutes/actinobacteria are designated ' + ', while proteobacterial arrangements are denoted ' - '. Here, note that all the bacteria grouped in ' + ' ('-') are not necessarily Gram-positive (-negative); for instance, Dictyoglomus thermophilum (phylum Dictyoglomi, grouped into ' + ') has been reported to stain Gram-negative (Saiki et al., 1985). Since the exceptional arrangements around Gtcc and lepA are only found in a few members of the Clostridia, they are not taken into account in the table. Although neighbouring gene pairs that are conserved among different genomes are known to interact physically at the protein level (Dandekar et al., 1998), such physical interactions seem unlikely among the gene products listed in Fig. 1. It is interesting to note that the patterns of division into ' + ' or ' - ' in Table 1 are almost consistent, suggesting an evolutionary event. Thus, bacterial phyla appear to reveal a fundamental split into two groups (superphyla): one comprises the Firmicutes, Synergistetes, Actinobacteria, Thermotogae, Chloroflexi, Deinococcus-Thermus, Fusobacteria and Dictyoglomi and the other consists of the Proteobacteria, Aquificae, Planctomycetes, Spirochaetes, Bacteroidetes, Chlorobi, Acidobacteria, Verrucomicrobia, termite group 1 and Nitrospirae ( $T$. yellowstonii). It is to be noted that an identical distribution of '-' is observed between T. yellowstonii and the Proteobacteria, suggesting their close evolutionary relationship.

\section{Conserved gene-pair analysis}

In order to assess evolutionary relationships of T. yellowstonii to other phyla at the level of overall genome organization, a conserved gene-pair analysis (Wolf et al., 2001; Korbel et al., 2002) was performed. Here, a conserved gene pair is defined as a pair of genes that are adjacent to each other in the same order on both genomes under comparison. For the sake of ready comparison of genome organization, we have generated reduced gene orders in which genes shared by the two genomes under pairwise comparison were retained, while genes present in only one genome were neglected. Using gene orders generated in this way, the fraction $f_{\mathrm{c}}$ of conserved gene pairs was evaluated in comparisons of T. yellowstonii to other bacteria. Values of $f_{\mathrm{c}}$ averaged over each phylum are listed in Table 2. The Mollicutes (Firmicutes) were neglected in this evaluation because of the extreme smallness of their genomes. As can be seen in Table 2, the genome organization of T. yellowstonii is most similar to those of the Deltaproteobacteria (0.238), followed by the Acidobacteria 
(a)

Thermotogae (7/7)

Thermotoga maritima

Thermosipho melanesiensis

Dictyoglomi (1/1)

Dictyoglomus thermophilum

Deinococcus-Thermus (3/3)

Deinococcus geothermalis

Thermus thermophilus

Fusobacteria (1/1)

Fusobacterium nucleatum

Actinobacteria (36/40)

Rubrobacter xylanophilus

Corynebacterium efficiens

Synergistetes (1/2)

Syntrophomonas wolfei

Firmicutes - Clostridia (19/24)

Symbiobacterium thermophilum

Pelotomaculum thermopropionicum

Clostridium acetobutylicum [4]

Heliobacterium modesticaldum

Firmicutes - Bacillales (9/23)

Bacillus halodurans [3]

Bacillus halodurans [3]

Firmicutes - Mollicutes (4/18)

Acholeplasma laidlawii

Aquificae (3/3)

'Aquifex aeolicus'

Sulfurihydrogenibium sp.

Nitrospirae (1/1)

Thermodesulfovibrio yellowstonii

Acidobacteria (1/2)

Acidobacteria bacterium

Planctomycetes (1/1)

Rhodopirellula baltica

Bacteroidetes (10/12)

Bacteroides thetaiotaomicron [2]

Cytophaga hutchinsonii

Termite group $1(1 / 1)$

'Elusimicrobium minutum'

'Coprothermobacter' (1/1)

Coprothermobacter proteolyticus

Proteobacteria - Deltaproteobacteria (18/19) Geobacter sulfurreducens

Proteobacteria - Epsilonproteobacteria (13/13) Campylobacter jejuni

Proteobacteria - Alphaproteobacteria (48/66)

Parvibaculum lavamentivorans

Proteobacteria - Betaproteobacteria (33/33) Neisseria meningitidis

Proteobacteria - Gammaproteobacteria (79/80)

Haemophilus influenzae

Exceptions in Firmicutes - Clostridia

Finegoldia magna

Alkaliphilus metalliredigens [5]

Alkaliphilus oremlandii [4]

Alkaliphilus oremlandii [4]

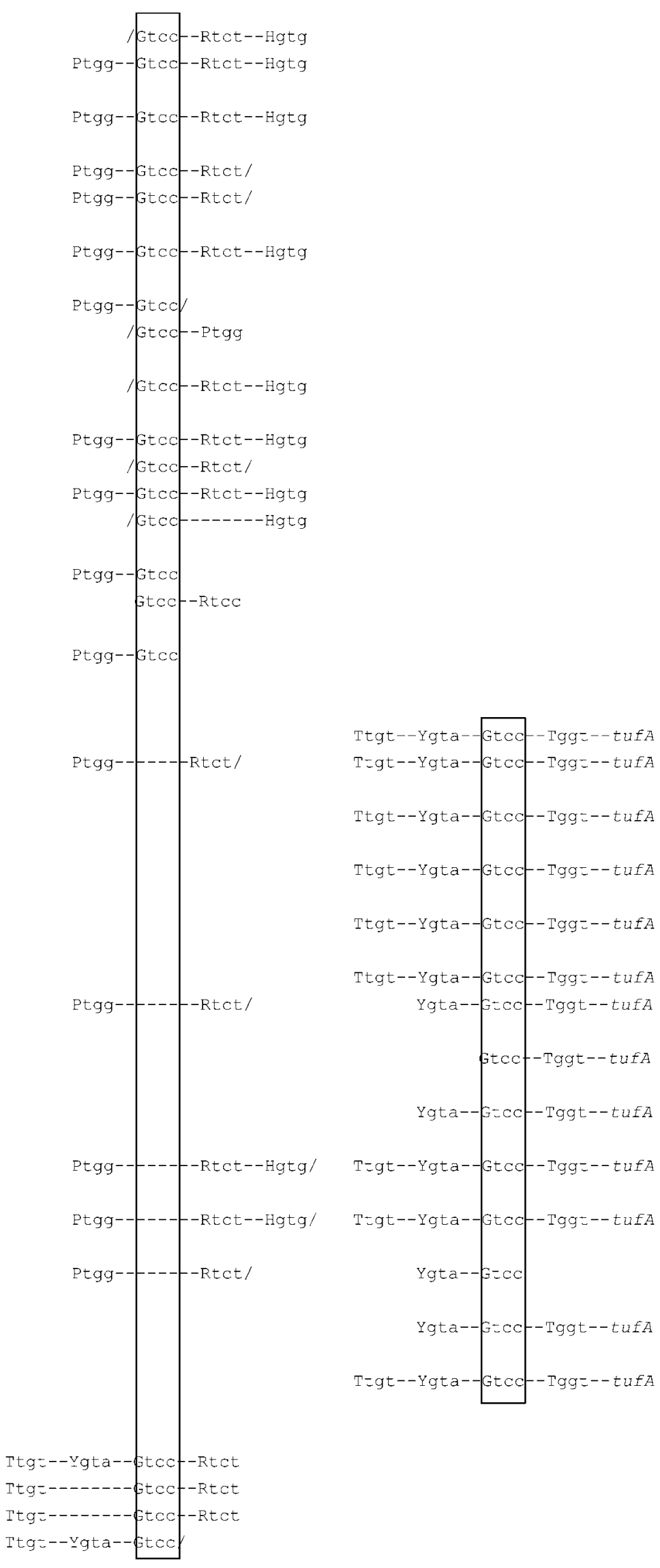

http://ijs.sgmjournals.org 
(b)

Thermotogae (7/7)

Thermotoga maritima

Dictyoglomi (1/1)

Dictyoglomus thermophilum

Chloroflexi (1/6)

Herpetosiphon aurantiacus

Deinococcus-Thermus (3/3)

Thermus thermophilus

Fusobacteria (1/1)

Fusobacterium nucleatum

Actinobacteria (39/40)

Rubrobacter xylanophilus

Synergistetes (2)

Syntrophomonas wolfei

Firmicutes - Clostridia (24/24)

Symbiobacterium thermophilum

Firmicutes - Bacillales (23/23)

Listeria monocytogenes

Firmicutes - Lactobacillales (27/27)

Lactobacillus plantarum

Firmicutes - Mollicutes (8/18)

Mycoplasma genitalium

Acholeplasma laidlawii

Aquificae (2/3)

'Aquifex aeolicus'

Nitrospirae (1/1)

Thermodesulfovibrio yellowstonii

Acidobacteria (2/2)

Acidobacteria bacterium

Spirochaetes (2/10)

Leptospira interrogans

Verrucomicrobia (3/3)

Opitutus terrae

Proteobacteria - Deltaproteobacteria (18/19) Geobacter sulfurreducens

Proteobacteria - Epsilonproteobacteria (13/13) Campylobacter jejuni

Proteobacteria - Alphaproteobacteria (42/66) Mesorhizobium loti

Proteobacteria - Betaproteobacteria (33) Neisseria meningitidis

Proteobacteria - Gammaproteobacteria (72/80)

Haemophilus influenzae

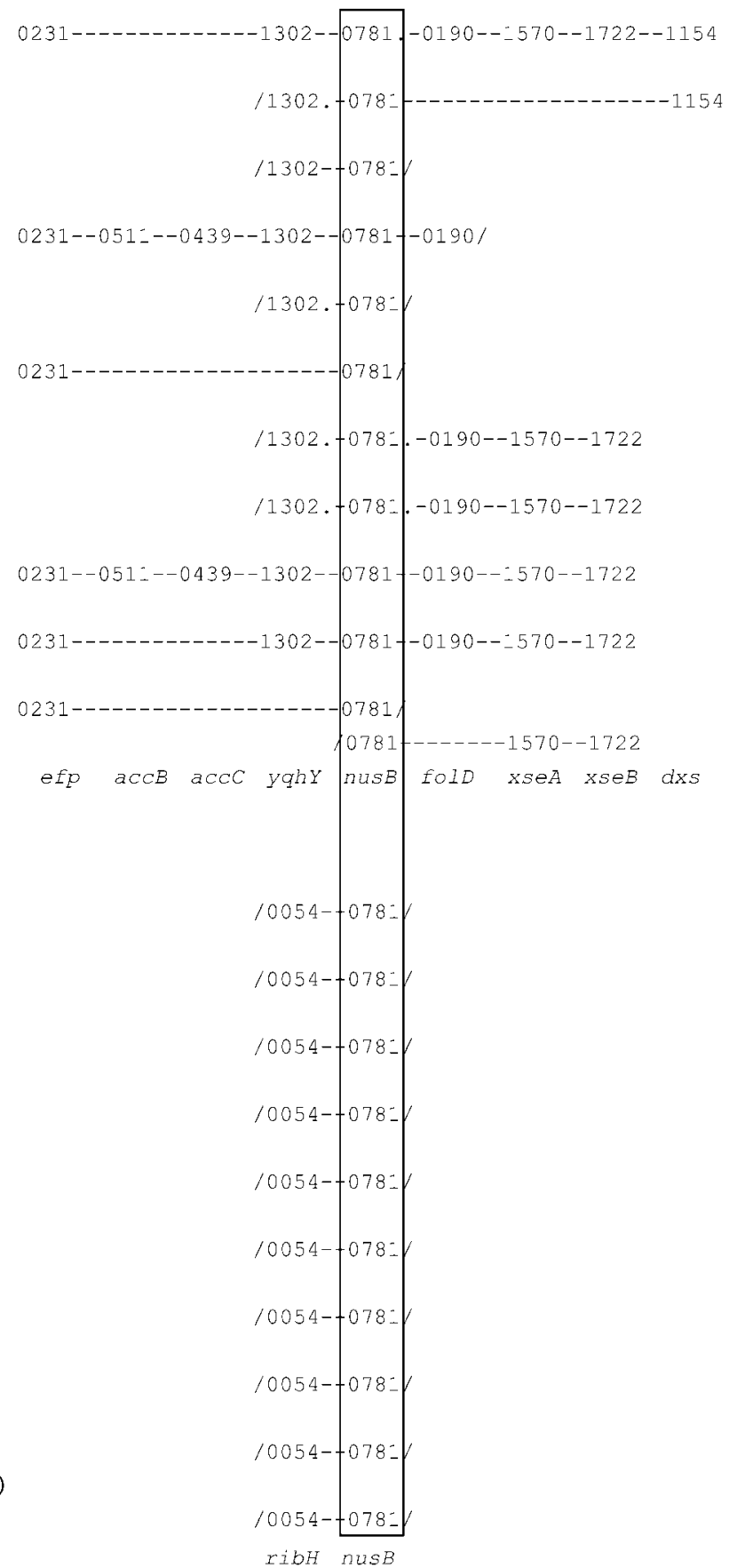

(0.217). Table 2 also lists the mean fraction of shared genes, $f_{\mathrm{s}}$. The gene content of T. yellowstonii is most similar to those of the Aquificae $\left(f_{s}=0.835\right)$, whereas no close similarity at the sequence level was found between them in BLAST analyses, as discussed below. Therefore, the similarity in gene content might represent thermophilic properties of those bacteria rather than their phylogenetic relationship.

\section{Identification of gene arrangements that suggest a close phylogenetic relationship of $T$. yellowstonii to the Proteobacteria}

My computer search has identified the following three gene arrangements, each of which suggests a gene translocation that has occurred in a common ancestor of $T$. yellowstonii and the Proteobacteria exclusive of other phyla. 
(c)

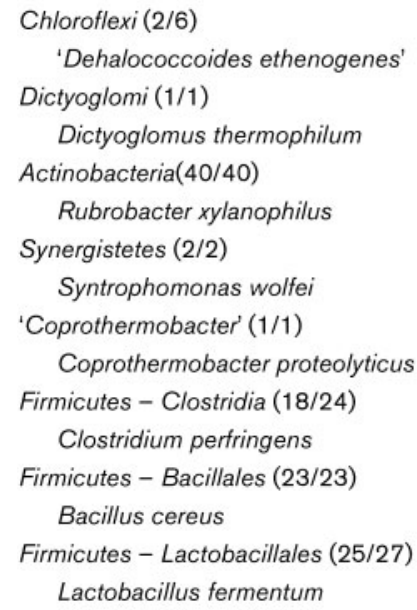

Aquificales (1/3)
Sulfurihydrogenibium sp.
Nitrospirae (1/1)
Thermodesulfovibrio yellowstonii
Planctomycetes (1/1)
Rhodopirellula baltica
Acidobacteria (1/2)
'Solibacter usitatus'
Spirochaetes (6/10)
Borrelia burgdorferi
Termite group 1 (1/1)
'Elusimicrobium minutum'
Proteobacteria/Delta (15/19)
Geobacter sulfurreducens
Sorangium cellulosum
Proteobacteria - Alphaproteobacteria (39/66)
Mesorhizobium loti
Proteobacteria - Betaproteobacteria (29/33)
Chromobacterium violaceum
Proteobacteria - Gammaproteobacteria (62/8
Xylella fastidiosa

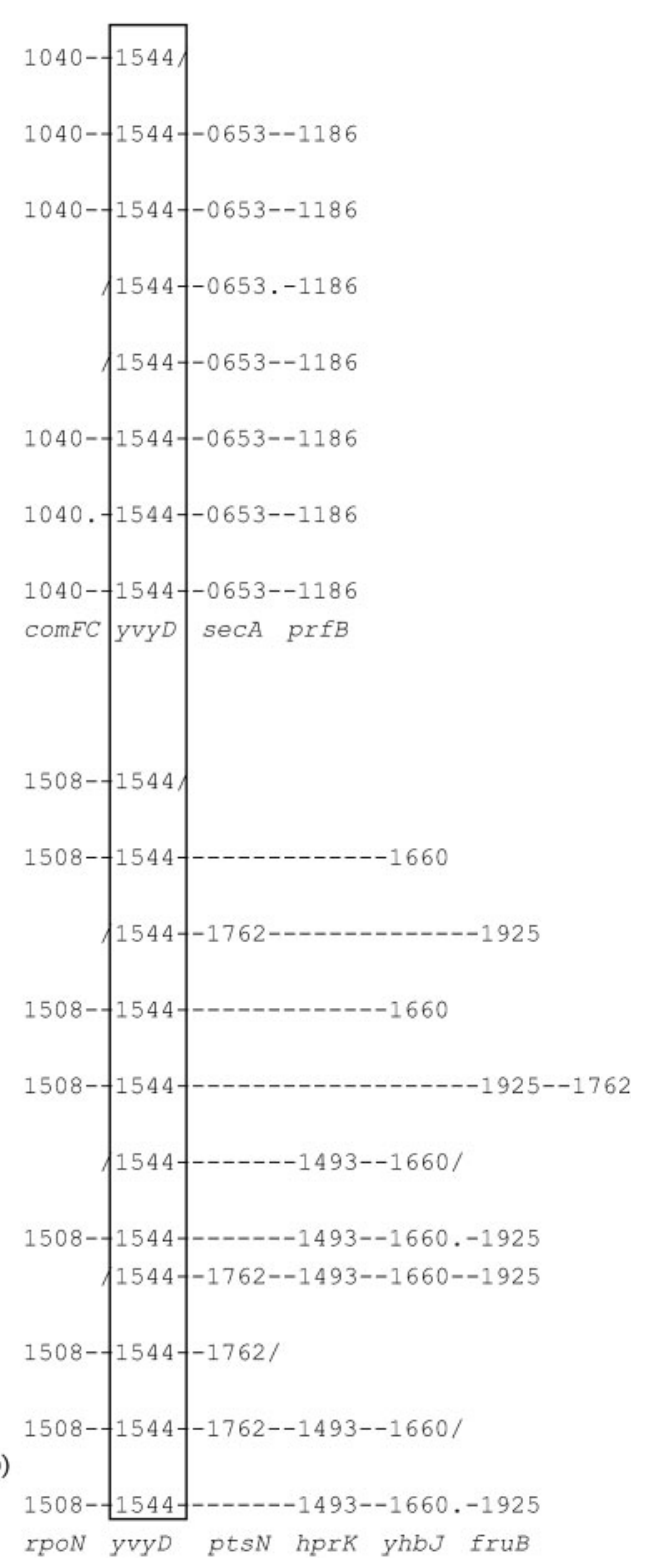

The asd gene (COG0136) coding for aspartate-semialdehyde dehydrogenase is located in the neighbourhood of dapB (COG0289), lysC (COG0527) and dapA (COG0329) in most members of superphylum 1, i.e. the Thermotogae, Dictyoglomi, Chloroflexi, Actinobacteria, Synergistetes and Firmicutes, and three members of superphylum 2, Acidobacteria, Chlamydiae and termite group 1. In contrast, in T. yellowstonii and the Proteobacteria, asd is adjacent to leuD (COG0066) and leuB (0473), as shown in Fig. 2(a). On the basis of these arrangements, it is likely that the arrangement dapB-asd-lysC (or other similar arrangements) was ancestral, and that the other arrangement leuD-leuB-asd was derived during evolution by a gene translocation of asd in a common ancestor of T. yellow- stonii and the Proteobacteria exclusive of the Thermotogae, Dictyoglomi, Chloroflexi, Actinobacteria, Synergistetes, Firmicutes, Acidobacteria, Chlamydiae and termite group 1. The common ancestry of $T$. yellowstonii and the Proteobacteria thus suggested implies their close evolutionary relationship.

Similarly, another translocation of the $g \operatorname{lm} U$ gene (COG1207) encoding $\mathrm{N}$-acetylglucosamine-1-phosphate uridyltransferase is likely to have occurred in a common ancestor of T. yellowstonii and the Proteobacteria; $g \operatorname{lm} U$ is located between atpD (COG0055)-atpC (COG0355) and $\operatorname{glmS}$ (COG0449)-COG2928 in T. yellowstonii and the Proteobacteria while, in most members of superphylum 1 
(d)

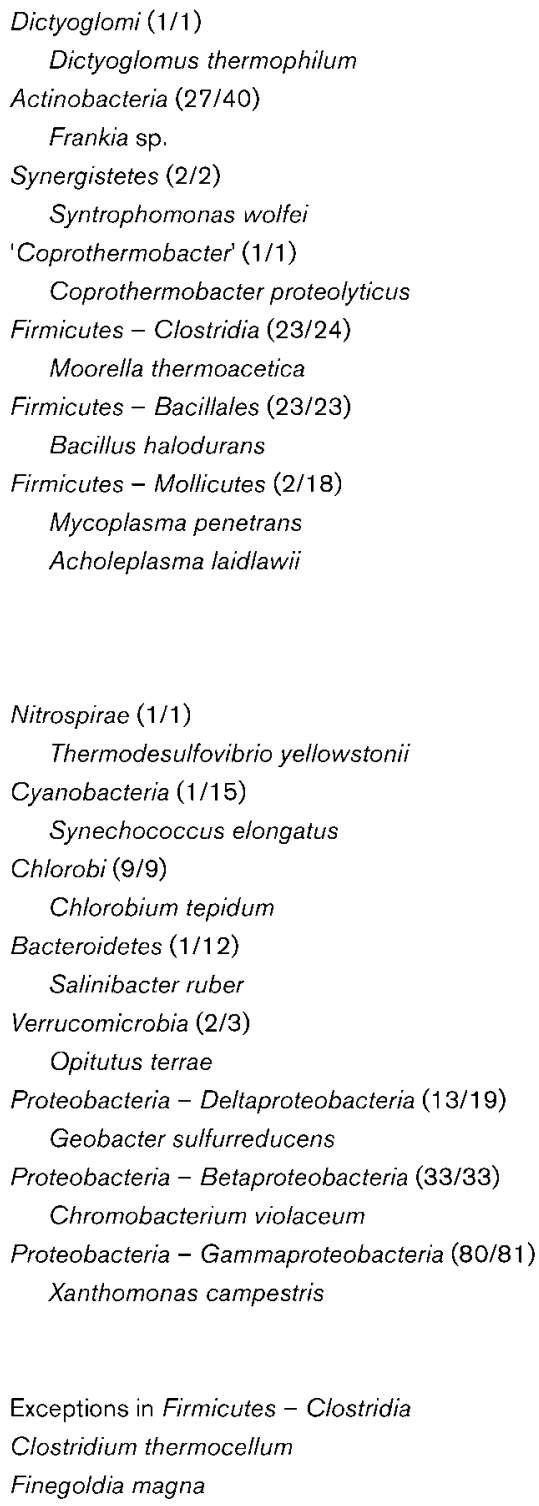

Fig. 1. Alternative gene arrangements around a glycine-tRNA gene with the anticodon sequence TCC (Gtcc) (a), nus $B$ (COG0781) (b), yvyD (COG1544) (c) and lepA (COG0481) (d). Protein-encoding genes are represented by four-digit COG numbers and genes encoding tRNAs are designated using the one-letter symbol for the cognate amino acid and the anticodon sequence in lower-case letters. Homologous genes are aligned vertically. Contiguous genes are connected by dashes, while split segments are interrupted by slashes. Genes with an asterisk are encoded on the complementary strand, and are transcribed from right to left; other genes are transcribed from left to right. For maximum alignment, some ORFs are represented by dots. The numerator in parentheses following the phylum name represents the number of genomes showing conserved gene arrangements and the denominator is the total number of compared genomes belonging to that phylum. Gene copy number per genome is shown in square brackets when greater than one.

(i.e. Thermotogae, Dictyoglomi, Chloroflexi, Actinobacteria, Synergistetes, Firmicutes and 'Coprothermobacter') and three phyla belonging to superphylum 2 (Planctomycetes, Spirochaetes and termite group 1), glmU is located between ispA (COG1947)-(Qttg) and prsA (COG0462) (Fig. 2b).
The third example can be observed in gene arrangements around psd (COG0688) and pssA (COG1183), respectively encoding phosphatidylserine decarboxylase and phosphatidylserine synthase. The two-gene cluster is located adjacent to moeA (COG0303) or $\operatorname{dedA}$ (COG0568) in the 
Table 1. Dichotomy of major bacterial phyla based on gene arrangements

+, Arrangement similar to the Firmicutes/Actinobacteria; -, proteobacterial arrangement. NA, No data available.

\begin{tabular}{|c|c|c|c|c|}
\hline \multirow[t]{2}{*}{ Phylum } & \multicolumn{4}{|c|}{ Gene arrangement } \\
\hline & Gtcc & nusB & $y v y D$ & lepA \\
\hline \multicolumn{5}{|l|}{ Superphylum 1} \\
\hline Firmicutes & + & + & + & + \\
\hline Synergistetes & + & + & + & + \\
\hline Actinobacteria & + & + & + & + \\
\hline Thermotogae & + & + & & \\
\hline Chloroflexi & + & + & + & \\
\hline Deinococcus-Thermus & + & + & & \\
\hline Fusobacteria & + & + & & \\
\hline Dictyoglomi & + & + & + & + \\
\hline 'Coprothermobacter' & - & NA & + & + \\
\hline \multicolumn{5}{|l|}{ Superphylum 2} \\
\hline Proteobacteria & - & - & - & - \\
\hline Aquificales & - & - & - & \\
\hline Planctomycetes & - & & - & \\
\hline Spirochaetes & & - & - & \\
\hline Bacteroidetes & - & & & - \\
\hline Chlorobi & & & & - \\
\hline Acidobacteria & - & - & - & \\
\hline Verrucomicrobia & & - & & - \\
\hline Termite group 1 & - & & - & \\
\hline Nitrospirae & - & - & - & - \\
\hline Cyanobacteria & & & & - \\
\hline
\end{tabular}

Actinobacteria, Synergistetes, Firmicutes, Spirochaetes and Verrucomicrobia, as shown in Fig. 2(c). In T. yellowstonii and Proteobacteria, the cluster is instead found between $i l v C$ (COG0059) and leuA (COG0119), suggesting a translocation of the $p s d-p s s A$ cluster in a common ancestor of T. yellowstonii and the Proteobacteria after the divergence of the Actinobacteria, Synergistetes, Firmicutes, Spirochaetes and Verrucomicrobia.

Combining these results, a common ancestor of $T$. yellowstonii and the Proteobacteria is inferred to have branched off from the trunk of superphylum 2 comprising the Acidobacteria, Chlamydiae, termite group 1, Planctomycetes, Spirochaetes and Verrucomicrobia, after the divergence of superphylum 1 . In this way, the three sets of gene arrangements identified here provide empirical evidence of a close (probably closest) evolutionary relationship between T. yellowstonii and the Proteobacteria.

\section{DISCUSSION}

This study was undertaken to clarify the phylogenetic placement of $T$. yellowstonii at the phylum level on the basis of a new phylogenetic method using gene orders of completely sequenced genomes. I first showed that $T$. yellowstonii shares four gene arrangements characteristic of
Table 2. Fractions of conserved gene pairs and shared genes in comparison with $T$. yellowstonii

The number of analysed genomes belonging to each phylum/class is shown in parentheses.

\begin{tabular}{|lcc|}
\hline Phylum & $f_{\mathbf{c}}$ & $f_{\mathbf{s}}$ \\
\hline Thermotogae (7) & 0.157 & 0.696 \\
Dictyoglomi (1) & 0.193 & 0.696 \\
Chloroflexi (6) & 0.177 & 0.619 \\
Deinococcus-Thermus (3) & 0.156 & 0.664 \\
Fusobacteria (1) & 0.153 & 0.570 \\
Actinobacteria (40) & 0.159 & 0.504 \\
Synergistetes (2) & 0.204 & 0.696 \\
Firmicutes & & \\
Clostridia (24) & 0.194 & 0.611 \\
Bacillales (22) & 0.167 & 0.531 \\
Lactobacillales (27) & 0.139 & 0.526 \\
Coprothermobacter (1) & 0.180 & 0.718 \\
Cyanobacteria (15) & 0.103 & 0.640 \\
Aquificae (3) & 0.143 & 0.835 \\
Acidobacteria (2) & 0.217 & 0.533 \\
Planctomycetes (1) & 0.150 & 0.523 \\
Chlorobi (9) & 0.164 & 0.764 \\
Bacteroidetes (12) & 0.160 & 0.573 \\
Spirochaetes (10) & 0.187 & 0.584 \\
Chlamydiae (6) & 0.161 & 0.574 \\
Verrucomicrobia (3) & 0.179 & 0.678 \\
Termite group 1 (1) & 0.174 & 0.703 \\
Proteobacteria & & \\
Deltaproteobacteria (19) & 0.238 & 0.656 \\
Epsilonproteobacteria (13) & 0.162 & 0.757 \\
Alphaproteobacteria (66) & 0.175 & 0.594 \\
Betaproteobacteria (32) & 0.211 & 0.590 \\
Gammaproteobacteria (80) & 0.196 & 0.568 \\
& & \\
\hline
\end{tabular}

the Proteobacteria, Aquificae, Planctomycetes, Spirochaetes, Bacteroidetes, Chlorobi, Acidobacteria, Verrucomicrobia and termite group 1, which form superphylum 2. The remaining phyla show a set of alternative gene arrangements and form superphylum 1 (Table 1). Second, the fraction of conserved gene pairs showed that the overall genome organization of $T$. yellowstonii is most similar to those of the Deltaproteobacteria (Table 2). Third, I have identified three arrangements that suggest gene translocations that are likely to have occurred in a common ancestor of T. yellowstonii and the Proteobacteria exclusive of virtually all other major bacterial phyla (Fig. 2), implying the closest evolutionary relationship between T. yellowstonii and the Proteobacteria. This conclusion is not surprising, since a close affinity of another genus, Nitrospira, belonging to the phylum Nitrospirae to the Deltaproteobacteria was suggested previously on the basis of 16S rRNA gene sequence comparison (Teske et al., 1994).

In order to assess evolutionary affinities of T. yellowstonii to other bacterial phyla at the protein sequence level, a BLAST best-hit analysis (Altschul et al., 1997) was performed (the results are provided in Supplementary Table S2). 
(a)

\section{Thermotogae}

Thermotoga maritima

Fervidobacterium nodosum

Dictyoglomi

Dictyoglomus thermophilum

Chloroflexi

'Dehalococcoides ethenogenes'

Roseiflexus castenholzi

Actinobacteria

Rubrobacter xylanophilus

Mycobacterium tuberculosis

Synergistetes

Syntrophomonas wolfei

Caldicellulosiruptor saccharolyticus

Firmicutes - Clostridia

Symbiobacterium thermophilum

Clostridium perfringens

Firmicutes - Bacillales

Bacillus subtilis

Listeria monocytogenes

Staphylococcus aureus

Exiguobacterium sibiricum

Firmicutes - Lactobacillales

Lactobacillus fermentum

Oenococcus oeni

Firmicutes - Mollicutes

Acholeplasma laidlawii

Acidobacteria

Acidobacteria bacterium

'Solibacter usitatus'

Chlamydiae

Chlamydophila felis

Chlamydia pneumoniae

Termite group 1

'Elusimicrobium minutum'

Nitrospirae

Thermodesulfovibrio yellowstonii

Proteobacteria - Deltaproteobacteria

Geobacter sulfurreducens

Geobacter lovleyi

Pelobacter propionicus

Syntrophobacter fumaroxidans

Proteobacteria - Alphaproteobacteria

Parvibaculum lavamentivorans

Brucella melitensis

Ehrlichia ruminantium

Proteobacteria - Betaproteobacteria

Ralstonia solanacearum

Methylobacillus flagellatus

Proteobacteria - Gammaproteobacteria

Pseudomonas syringae

Marinomonas sp.

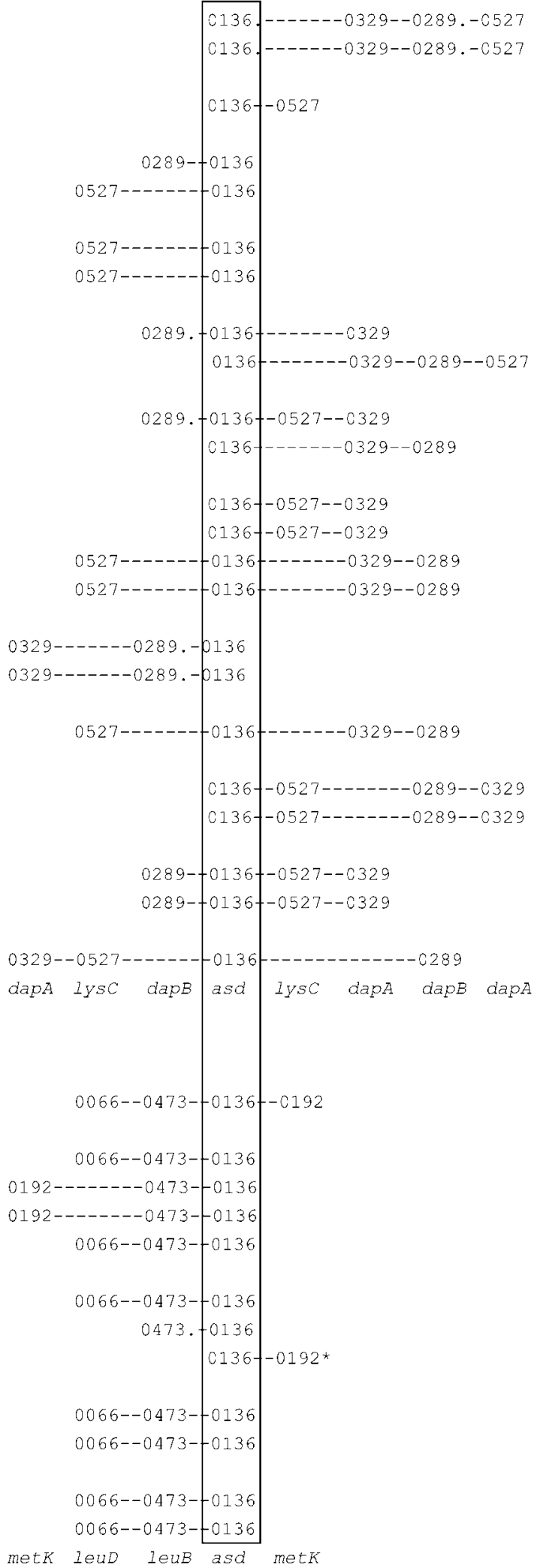


(b)

\author{
Thermotogae \\ Thermotoga maritima \\ Fervidobacterium nodosum \\ Dictyoglomi \\ Dictyoglomus thermophilum [2] \\ Chloroflexi \\ Roseiflexus castenholzii \\ Actinobacteria \\ Rubrobacter xylanophilus \\ Arthrobacter aurescens \\ Synergistetes \\ Syntrophomonas wolfei \\ Firmicutes - Clostridia \\ Symbiobacterium thermophilum \\ Moorella thermoacetica \\ Firmicutes - Bacillales \\ Bacillus subtilis \\ Staphylococcus aureus \\ Firmicutes - Lactobacillales \\ Lactobacillus sakei \\ Pediococcus pentosaceus \\ 'Coprothermobacter' \\ Coprothermobacter proteolyticus \\ Planctomycetes \\ Rhodopirellula baltica \\ Spirochaetes \\ Leptospira interrogans \\ Leptospira biflexa \\ Termite group 1 \\ 'Elusimicrobium minutum'
}

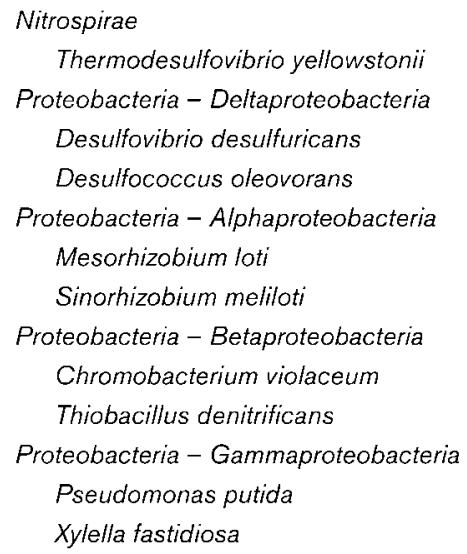

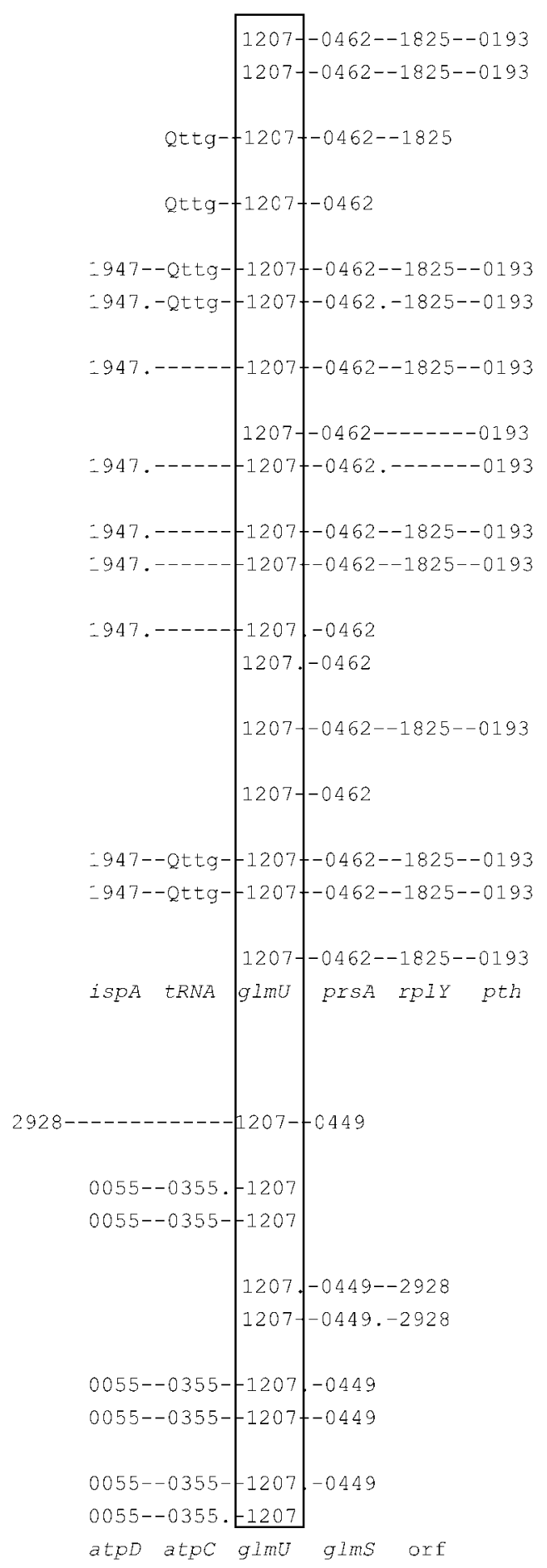

Sequence data of 52 ribosomal proteins from T. yellowstonii were used as the query sequences. Since ribosomal proteins function as components of a large macromolecular complex, their genes are unlikely to undergo lateral gene transfer with a high probability (Jain et al., 1999). The best- hit sequence was searched for against the KEGG GENES database, which collects and stores sequences translated from completely sequenced genomes and is available at GenomeNet (http://www.genome.ad.jp/). The BLAST analysis showed phylogenetic affinities of T. yellowstonii both to 
(c)

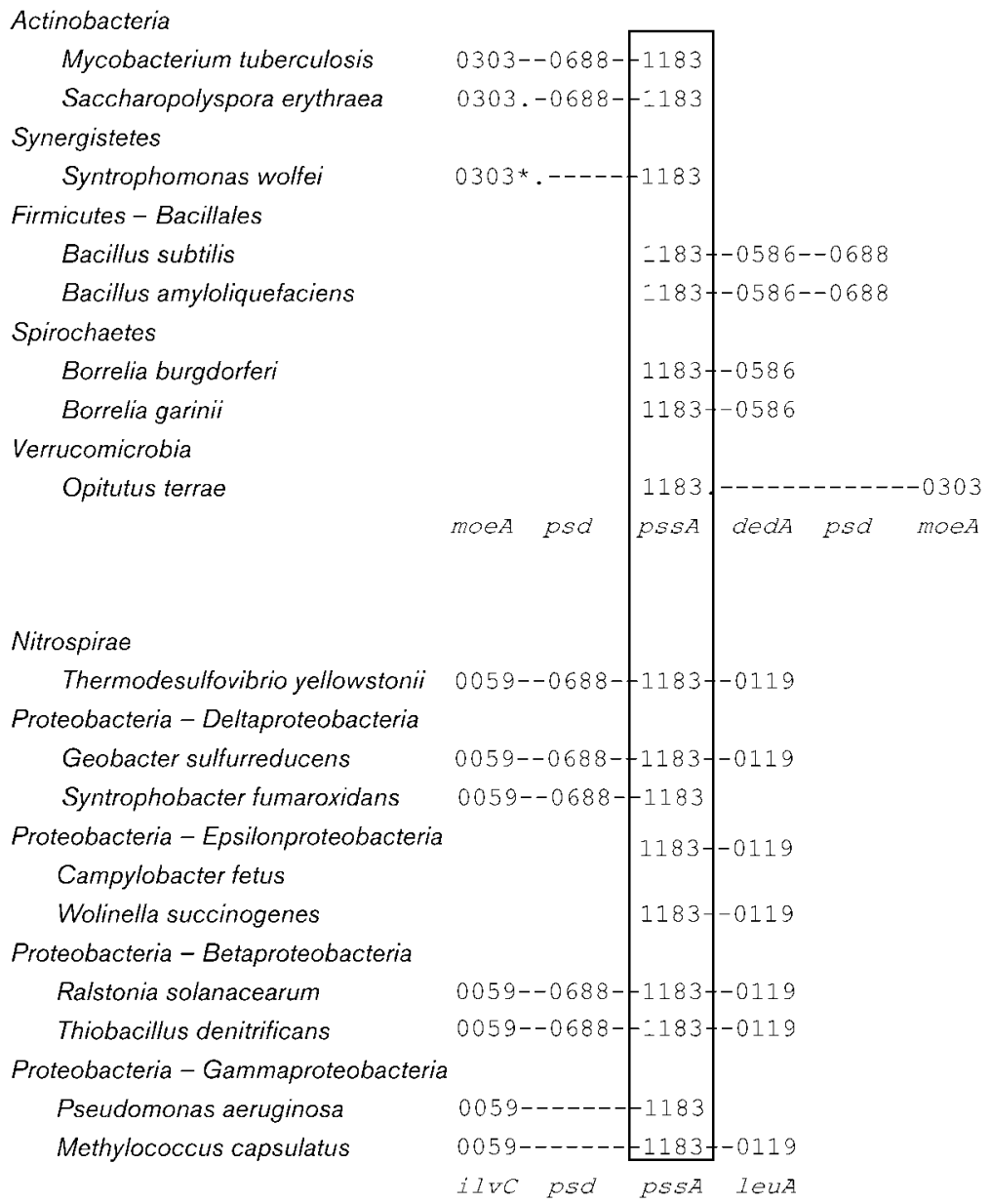

Fig. 2. Gene translocations that are likely to have occurred in a common ancestor of T. yellowstonii and the Proteobacteria. Transposed genes are boxed. (a) asd (COG0136), (b) glmU (COG1207) and (c) psd (COG0688)-pssA (COG1183).

the Clostridia (Firmicutes) and to the Proteobacteria: 18 best-hit sequences from the Clostridia, 17 best hits from the Proteobacteria and three best hits from the Synergistetes, Thermotogae, Cyanobacteria and Dictyoglomi. Thus, the close phylogenetic affinity of T. yellowstonii to the Proteobacteria obtained from gene arrangement analyses is compatible with amino acid sequence comparisons, although the Clostridia are also assigned as another close relative. This assignment of the Clostridia seems to represent a limitation inherent in the BLAST best-hit analysis; it is known that genes that appear to be the most similar based on BLAST hits are often not phylogenetically the closest relatives (Koski \& Golding, 2001).

The gene arrangements compared in Fig. 1 suggest a dichotomic division of bacterial phyla into two groups, superphyla 1 and 2. Table 1 shows a consistent grouping of the major phyla except for 'Coprothermobacter'. A similar partition of bacterial phyla has been reported on the basis of domain architectures of RNA polymerase subunits RpoB, RpoC and RpoD; three distinct large inserts of more than 100 amino acids are shared by the Proteobacteria, Aquificae, Chlamydiae, Spirochaetes, Chlorobi and Planctomycetes, while the Firmicutes, Actinobacteria, Cyanobacteria, Thermus-Deinococcus, Thermotogae and Chloroflexi all lack these inserts (Gupta, 2003; Iyer et al., 2004). Thus, these two patterns are almost identical except for the Cyanobacteria, which are included in superphylum 2 by the sole criterion of the gene arrangements around lepA (see Table 1). Furthermore, these partitions of bacterial phyla into two groups are generally compatible with the topologies of genome trees of concatenated protein sequences (Iyer et al., 2004; Wu \& Eisen, 2008). A genome tree reconstructed quite recently using a concatenated dataset of 31 housekeeping proteins also confirms this grouping (Ward et al., 2009); the Proteobacteria, Acidobacteria, Chlorobi, Bacteroidetes, Spirochaetes and Chlamydiae form a distinct clade with a 
high bootstrap probability of $81 \%$, while its sister clade comprises the Actinobacteria, Firmicutes, Fusobacteria, Chloroflexi, Cyanobacteria, Thermotogae, Aquificae and Deinococcus-Thermus. These patterns of congruence strongly suggest that the divisions reflect a fundamental split in bacterial evolution rather than lateral gene transfers within each superphylum. It may be reasonable to consider that those phyla positioned near the boundary of the two superphyla tend to be grouped differently depending on which translocations or insertions are used as the criteria of partition. The inconsistencies found for the Cyanobacteria and for 'Coprothermobacter' might be understood in this way. Although a close evolutionary relationship between the Chlamydiae and Verrucomicrobia has been proposed (Wagner \& Horn, 2006; Griffiths \& Gupta, 2007), the present gene arrangement analyses provided no information about the phylogenetic placement of the Chlamydiae. The phylogenetic placement of the Aquificae remains puzzling. Gene-order, gene-content and indel analyses all suggest a late emergence of this phylum (Wolf et al., 2001; Korbel et al., 2002; Iyer et al., 2004; Griffiths \& Gupta, 2004; Kunisawa, 2006), while phylogenetic trees based on the 16S rRNA gene and concatenated protein sequences reveal a basal early branching phylum (Hugenholtz et al., 1998; Wolf et al., 2001; Coenye \& Vandamme, 2004; Ciccarelli et al., 2006; Wu \& Eisen, 2008; Ward et al., 2009). In any case, the close evolutionary relationship between T. yellowstonii and the Proteobacteria indicates that thermophily is likely to have been acquired on some branches of bacterial evolution.

\section{ACKNOWLEDGEMENTS}

The author would like to thank the anonymous referees for helpful suggestions and comments.

\section{REFERENCES}

Achenbach-Richter, L., Gupta, R., Stetter, K. O. \& Woese, C. R. (1987). Were the original eubacteria thermophiles? Syst Appl Microbiol 9, 34-39.

Altschul, S. F., Madden, T. L., Schaffer, A. A., Zhang, J., Zhang, Z., Miller, W. \& Lipman, D. J. (1997). Gapped BLAST and PSI-BLAST: a new generation of protein database search programs. Nucleic Acids Res $\mathbf{2 5}$ 3389-3402.

Brown, J. R., Douady, C. J., Italia, M. J., Marshall, W. E. \& Stanhope, M. J. (2001). Universal trees based on large combined protein sequence data sets. Nat Genet 28, 281-285.

Castro, H. F., Williams, N. H. \& Ogram, A. (2000). Phylogeny of sulfate-reducing bacteria. FEMS Microbiol Ecol 31, 1-9.

Ciccarelli, F. D., Doerks, T., von Mering, C., Creevey, C. J., Snel, B. \& Bork, P. (2006). Toward automatic reconstruction of a highly resolved tree of life. Science 311, 1283-1287.

Coenye, T. \& Vandamme, P. (2004). A genomic perspective on the relationship between the Aquificales and the epsilon-Proteobacteria. Syst Appl Microbiol 27, 313-322.
Dandekar, T., Snel, B., Huynen, M. \& Bork, P. (1998). Conservation of gene order: a fingerprint of proteins that physically interact. Trends Biochem Sci 23, 324-328.

Daubin, V., Gouy, M. \& Perriere, G. (2002). A phylogenomic approach to bacterial phylogeny: evidence of a core of genes sharing a common history. Genome Res 12, 1080-1090.

Delsuc, F., Brinkmann, H. \& Philippe, H. (2005). Phylogenomics and the reconstruction of the tree of life. Nat Rev Genet 6, 361-375.

Etchebehere, C., Pavan, M. E., Zorzópulos, J., Soubes, M. \& Muxí, L. (1998). Coprothermobacter platensis sp. nov., a new anaerobic proteolytic thermophilic bacterium isolated from an anaerobic mesophilic sludge. Int J Syst Bacteriol 48, 1297-1304.

Griffiths, E. \& Gupta, R. S. (2004). Signature sequences in diverse proteins provide evidence for the late divergence of the order Aquificales. Int Microbiol 7, 41-52.

Griffiths, E. \& Gupta, R. S. (2007). Phylogeny and shared conserved inserts in proteins provide evidence that Verrucomicrobia are the closest known free-living relatives of chlamydiae. Microbiology 153, 2648-2654.

Gupta, R. S. (1998). Protein phylogenies and signature sequences: a reappraisal of evolutionary relationships among archaebacteria, eubacteria and eukaryotes. Microbiol Mol Biol Rev 62, 1435-1491.

Gupta, R. S. (2003). Evolutionary relationships among photosynthetic bacteria. Photosynth Res 76, 173-183.

Gupta, R. S. \& Griffiths, E. (2002). Critical issues in bacterial phylogenies. Theor Popul Biol 61, 423-434.

Henry, E. A., Devereux, R., Maki, J. S., Gilmour, C. C., Woese, C. R., Mandelco, L., Schauder, R., Remsen, C. C. \& Mitchell, R. (1994). Characterization of a new thermophilic sulfate-reducing bacterium Thermodesulfovibrio yellowstonii, gen. nov. and sp. nov.: its phylogenetic relationship to Thermodesulfobacterium commune and their origins deep within the bacterial domain. Arch Microbiol 161, 6269.

Hugenholtz, P., Goebel, B. M. \& Pace, N. R. (1998). Impact of culture-independent studies on the emerging phylogenetic view of bacterial diversity. J Bacteriol 180, 4765-4774.

Iyer, L. M., Koonin, E. V. \& Aravind, L. (2004). Evolution of bacterial RNA polymerase: implications for large-scale bacterial phylogeny, domain accretion, and horizontal gene transfer. Gene 335, 73-88.

Jain, R., Rivera, M. C. \& Lake, J. A. (1999). Horizontal gene transfer among genomes: the complexity hypothesis. Proc Natl Acad Sci U S A 96, 3801-3806.

Korbel, J. O., Snel, B., Huynen, M. A. \& Bork, P. (2002). SHOT: a web server for the construction of genome phylogenies. Trends Genet 18, $158-162$.

Koski, L. B. \& Golding, G. B. (2001). The closest BLAST hit is often not the nearest neighbor. J Mol Evol 52, 540-542.

Kunisawa, T. (2001). Gene arrangements and phylogeny in the class Proteobacteria. J Theor Biol 213, 9-19.

Kunisawa, T. (2003). Gene arrangements and branching orders of Gram-positive bacteria. J Theor Biol 222, 495-503.

Kunisawa, T. (2006). Dichotomy of major bacterial phyla inferred from gene arrangement comparisons. J Theor Biol 239, 367-375.

Lowe, T. M. \& Eddy, S. R. (1997). tRNAscan-SE: a program for improved detection of transfer RNA genes in genomic sequence. Nucleic Acids Res 25, 955-964.

Pearson, W. R. \& Lipman, D. J. (1988). Improved tools for biological sequence comparison. Proc Natl Acad Sci U S A 85, 2444-2448.

Saiki, T., Kobayashi, Y., Kawagoe, K. \& Beppu, T. (1985). Dictyoglomus thermophilum gen. nov., sp. nov., a chemoorganotrophic, anaerobic, thermophilic bacterium. Int J Syst Bacteriol 35, 253-259. 
Sankoff, D., Leduc, G., Antoine, N., Paquin, B., Lang, B. F. \& Cedergren, R. (1992). Gene order comparisons for phylogenetic inference: evolution of the mitochondrial genome. Proc Natl Acad Sci U S A 89, 6575-6579.

Tatusov, R. L., Koonin, E. V. \& Lipman, D. J. (1997). A genomic perspective on protein families. Science 278, 631-637.

Tatusov, R. L., Natale, D. A., Garkavtsev, I. V., Tatusova, T. A., Shankavaram, U. T., Rao, B. S., Kiryutin, B., Galperin, M. Y., Fedorova, N. D. \& Koonin, E. V. (2001). The COG database: new developments in phylogenetic classification of proteins from complete genomes. Nucleic Acids Res 29, 22-28.

Teeling, H., Lombardot, T., Bauer, M., Ludwig, W. \& Glöckner, F. O. (2004). Evaluation of the phylogenetic position of the planctomycete 'Rhodopirellula baltica' SH 1 by means of concatenated ribosomal protein sequences, DNA-directed RNA polymerase subunit sequences and whole genome trees. Int J Syst Evol Microbiol 54, 791-801.
Teske, A., Alm, E., Regan, J. M., Toze, S., Rittmann, B. E. \& Stahl, D. A. (1994). Evolutionary relationships among ammonia- and nitrite-oxidizing bacteria. J Bacteriol 176, 6623-6630.

Wagner, M. \& Horn, M. (2006). The Planctomycetes, Verrucomicrobia, Chlamydiae and sister phyla comprise a superphylum with biotechnological and medical relevance. Curr Opin Biotechnol 17, 241-249.

Ward, N. L., Challacombe, J. F., Janssen, P. H., Henrissat, B., Coutinho, P. M., Wu, M., Xie, G., Haft, D. H., Sait, M. \& other authors (2009). Three genomes from the phylum Acidobacteria provide insight into their lifestyles in soils. Appl Environ Microbiol 75, 20462056.

Wolf, Y. I., Rogozin, I. B., Grishin, N. V., Tatusov, R. L. \& Koonin, E. V. (2001). Genome trees constructed using five different approaches suggest new major bacterial clades. BMC Evol Biol 1, 8 .

Wu, M. \& Eisen, J. A. (2008). A simple, fast, and accurate method of phylogenomic inference. Genome Biol 9, R151. 